\title{
TeV gamma-ray emission from gamma-ray bursts and ultra high energy cosmic rays ${ }^{\star}$
}

\section{T. Totani}

Theory Division, National Astronomical Observatory, Mitaka, Tokyo 181-8588, Japan

e-mail: totani@th.nao.ac.jp

Received December 1, 1999; accepted January 3, 2000

\begin{abstract}
Some recent experiments detecting very high energy (VHE) gamma-rays above $10-20 \mathrm{TeV}$, independently reported VHE bursts for some bright gamma-ray bursts (GRBs), although further confirmation is necessary. If these signals are truly from GRBs, these GRBs must emit a much larger amount of energy as VHE gammarays than in the ordinary photon energy range of GRBs $(\mathrm{keV}-\mathrm{MeV})$. We show that such extreme phenomena can be reasonably explained by synchrotron radiation of protons accelerated to $\sim 10^{20-21} \mathrm{eV}$. Protons must carry a much larger amount of energy than electrons, suggesting that energy transfer from protons into electrons in shocked matter is very inefficient. The total energy of GRBs becomes as large as $\gtrsim 10^{55}(\Delta \Omega / 4 \pi)$ ergs and hence a strong beaming of GRB emission is highly likely. There may also be $\mathrm{TeV}$ emission in afterglow phase from external shocks, and proton synchrotron in this phase gives a quantitative explanation for the famous long duration $\mathrm{GeV}$ emission from GRB 940217. Most TeV gamma-rays are absorbed in intergalactic space by interactions with the infrared and microwave background radiations, and finally form gamma-ray background in $\mathrm{GeV}$ range. This process may explain the extragalactic background radiation observed in the EGRET range. We also discuss absorption of $\mathrm{TeV}$ gamma-rays in the shocked region of GRBs by the $\gamma \gamma \rightarrow \mathrm{e}^{ \pm}$process, which may provide a new channel for energy transfer from protons into electrons (and positrons). This process should be important for the energetics of GRBs.
\end{abstract}

Key words: gamma-ray bursts (GRB)

Two major ground-based cosmic ray/gamma-ray detectors, the Tibet air shower array (Amenomori et al.

\footnotetext{
* This article had been submitted as a contribution paper in the "Rome '98 Gamma-Ray Burst workshop" and should have appeared in the A\&AS special issue for the workshop (A\&AS 1999, $138(3))$.
}

1996) and the HEGRA AIROBICC Cherenkov array (Padilla et al. 1998) have independently reported significant excesses of $10-20 \mathrm{TeV}$ gamma-rays coincident with some GRBs both in direction and burst time. The statistical significance was estimated to be about 6 sigma and 2.7 sigma for the Tibet and HEGRA groups, respectively. Both experiments suggest a time scale of $10 \mathrm{TeV}$ emission as $\sim 10$ seconds. Considering the fact that two different experimental groups independently reported similar significant signals of $10-20 \mathrm{TeV}$ gamma-rays, these observations are now worth theoretical interpretations. Although further observations are necessary to confirm these indications of VHE bursts, these VHE bursts, if confirmed, would result in a drastic change to our picture of $\mathrm{GRBs}^{1}$.

Such $\sim 10 \mathrm{TeV}$ gamma-rays are absorbed in the intergalactic field by $\mathrm{e}^{ \pm}$creation with the cosmic infrared background, and are visible only for neaby GRBs of $z \sim$ $0.1-0.2$. Taking into account the absorption, GRBs having the VHE emission must emit a much larger amount of energy as VHE gamma-rays than in the ordinary photon energy range of $\mathrm{keV}-\mathrm{MeV}$, by a factor of $\sim 10^{2}-10^{3}$ (Totani 1998b). However, such extreme phenomena can be explained if protons carry about $m_{\mathrm{p}} / m_{\mathrm{e}} \sim 2000$ times larger energy than electrons. Protons are responsible for $\mathrm{TeV}$ gamma-rays and electrons are for ordinary soft gamma-ray emission in $\mathrm{keV}-\mathrm{MeV}$. This assumption is not unreasonable because the origin of the GRB energy is kinetic energy of ultra-relativistic bulk motion (see, e.g., Piran 1997 for a review). If the energy transfer from protons into electrons is inefficient, protons carry much larger energy than electrons (Totani 1998a). Very large energy $\left(\sim 10^{55-56}(\Delta \Omega / 4 \pi)\right.$ erg) is required for the engine of GRBs, but it is not theoretically impossible if GRB emission is strongly beamed. The energy available

\footnotetext{
1 After the conference, the Milagro group reported an interesting excess of gamma-rays above a few hundreds $\mathrm{GeV}$ from GRB 970417a, with a chance probability of $1.510^{-3}$ (McEnery et al. 1999).
} 
by MHD-like processes is $\sim 10^{54}$ erg for mergers of compact objects or collapses of massive stars, and beaming of $(\Delta \Omega / 4 \pi) \sim 10^{-2}$ is not unreasonable. Furthermore, GRB 990123 after the conference had a large total energy of $\sim 310^{54}(\Delta \Omega / 4 \pi)$ erg (Kulkarni et al. 1999), suggesting that the true kinetic energy emission is reaching at least $\sim 10^{55}(\Delta \Omega / 4 \pi)$ erg.

We have shown that (Totani 1998b), in such energetic GRBs, protons can be accelerated to $10^{20-21} \mathrm{eV}$, which is the energy scale of ultra high energy cosmic rays (UHECRs), and proton synchrotron radiation of these UHE protons is just in $1-10 \mathrm{TeV}$ range. Since the cooling time scale of these UHE protons for an observer is as short as $\sim \mathrm{s}$ in the internal shocks, a considerable fraction of the total energy of relativistic motion can be extracted as VHE gamma-rays by protonsynchrotron radiation. We suggest that this process is responsible for the very strong $10 \mathrm{TeV}$ emission possibly detected by the Tibet and HEGRA groups. If this interpretation is correct, the indications of $\mathrm{TeV}$ bursts by the Tibet and HEGRA groups support the hypothesis that UHECRs observed on the Earth are produced by GRBs (Waxman 1995; Milgrom \& Usov 1995; Vietri 1995). The proton synchrotron radiation would exist also in the afterglow phase with the time scale of a few days, and this may be detected by Cerenkov $\mathrm{TeV}$ telescopes (Totani 1998a). The famous long duration $\mathrm{GeV}$ emission from GRB 940217 (Hurley et al. 1994) can be explained by the proton synchrotron in the $\mathrm{GeV}$ range in the early afterglow phase (Totani 1998a).

The other interesting consequence of our model is the possible contribution of GRBs to the extragalactic gamma-ray background radiation observed in the EGRET range (e.g., Sreekumar et al. 1998). In order to explain the energy density of the GeV background, energy production of $\sim 10^{55}(\Delta \Omega / 4 \pi)$ erg per one GRB is required, which is about $10^{3}$ higher than the conventional estimate of the energy of GRBs. However, in our model, protons carry 2000 times larger energy than electrons and most of the energy is radiated in $\mathrm{TeV}$ range by proton synchrotron. Most TeV gamma-rays from cosmological GRBs are absorbed in the intergalactic field by $\mathrm{e}^{ \pm}$-pair creation with the cosmic infrared background, and the pairs generate $\mathrm{GeV}$ gamma-rays by the inverse-Compton scattering of the CMB photons and subsequent cascades. Hence the energy emitted as TeV gamma-rays is eventually converted into $\mathrm{GeV}$ gamma-rays. Therefore, the shortage of the energy by a factor of $10^{3}$ is just compensated by the proton-electron mass ratio and hence GRBs can significantly contribute, or even explain the extragalactic GeV background flux (Totani 1999a). It must be investigated in future studies whether the spectrum of the GRB background predicted by this scenario is consistent with the observed spectrum.

We finally discuss the possibility that VHE gammarays are absorbed within the internal shocks by the $\gamma \gamma \rightarrow$ $\mathrm{e}^{ \pm}$reaction and hence cannot escape from GRBs to be observed (Baring \& Harding 1997; Böttcher \& Dermer 1998). The optical depth of this reaction in our model is estimated to be of order unity, and strongly dependent on the bulk Lorentz factor $\Gamma$ of GRBs as $\propto \Gamma^{5}$ (Totani 1999b). This suggests that, for some GRBs in which the optical depth is of order unity, the $\mathrm{e}^{ \pm}$-pair creation reaction gives an interesting new channel for energy transfer from protons into electrons (and positrons) in internal shocks. Therefore this reaction is very important for the energetics of GRBs. It can also be shown that the synchrotron radiation of the pairs is in $\sim \mathrm{MeV}$ range for observers, and hence we can attribute the ordinary sub-MeV band emission of GRBs to the synchrotron radiation of these $\mathrm{e}^{ \pm}$pairs created by absorption of $\mathrm{TeV}$ gamma-rays.

If this picture is true, the efficiency of conversion of kinetic energy into $\mathrm{MeV}$ photons depends on the efficiency of pair-creation. If the pair-creation reaction is efficient, we should observe very strong GRBs such as GRB 990123 with $E \gtrsim 10^{54}(\Delta \Omega / 4 \pi)$ erg. In this case the efficiency in the conversion of kinetic energy into $\mathrm{MeV}$ photons is determined only by that of acceleration of protons into UHE energies $\left(\sim 10^{20} \mathrm{eV}\right)$. On the other hand, if this reaction is inefficient, there is no channel of energy transfer from protons into electrons in the internal shock and hence we observe weak GRBs with $E \sim 10^{51}(\Delta \Omega / 4 \pi)$ erg by the synchrotron radiation of original electrons which carry a much smaller fraction of kinetic energy by the $m_{\mathrm{e}} / m_{\mathrm{p}}$ ratio.

Because of the strong dependence of the opacity of pair-creation on $\Gamma$ as mentioned above, a modest difference of $\Gamma$ from one GRB to another explains the wide dispersion (by a factor of more than 100) in the observed total energies of GRBs (Totani 1999b). A unified picture for the energetics of GRBs is then that all GRBs emit roughly the same amount of energy $\left(\sim 10^{55}(\Delta \Omega / 4 \pi)\right.$ erg $)$ as kinetic motion with the same beaming factor, and the apparent diversity of GRB luminosity is due to the different efficiency of sub-MeV gamma-ray production. This picture is consistent with the observational fact that there is almost no correlation between GRB luminosity and afterglow luminosity. If GRB luminosity is proportional to the true kinetic energy of relativistic outflow, we should observe a correlation between GRB and afterglow luminosities. However, roughly the same amount of kinetic energy is injected into interstellar medium regardless of the GRB luminosity in our scenario, and hence there should be no correlation between GRBs and afterglows as observed.

Acknowledgements. The author has been supported by the Research Fellowships of the Japan Society for the Promotion of Science for Young Scientists, and the Grant-in-Aid for the Scientific Research Fund (No. 3730) of the Ministry of Education, Science, and Culture of Japan. 


\section{References}

Amenomori M., et al., 1996, A\&A 311, 919

Baring M.G., Harding A.K., 1997, ApJ 491, 663

Böttcher M., Dermer C.D., 1998, ApJ 499, L131

Coppi P.S., Aharonian F.A., 1997, ApJ 487, L9

Hurley K., et al., 1994, Nat 372, 652

Kulkarni S., et al., 1999, Nat 398, 389

McEnery J.E., et al., 1999, astro-ph/9910549

Milgrom M., Usov V., 1995, ApJ 449, L37

Padilla L., et al., 1998, A\&AS 337, 43
Piran T., 1997, in Unsolved Problems in Astrophysics, Bahcall J.N. and Ostriker J.P. (eds.). New Jersey: Princeton University Press, p. 343

Sreekumar P., et al., 1998, ApJ 494, 523

Totani T., 1998a, ApJ 502, L13

Totani T., 1998b, ApJ 509, L81

Totani T., 1999a, Astropart. Phys. 11, 451

Totani T., 1999b, MNRAS 307, L41

Vietri M., 1995, ApJ 453, 883

Waxman E., 1995, Phys. Rev. Lett. 75, 386 